\title{
AMAURY GRIMAND
}

Université Nantes, IEA, LEMNA

EWAN OIRY

ESG-UQAM, Montréal, Québec

\section{AURÉLIEN RAGAIGNE}

Université de Poitiers, IAE, CEREGE

\section{Paradoxes, modes de régulation et perspectives théoriques}

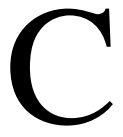

e dossier rassemble des articles présentant une réflexion sur le management des paradoxes. Ils ont en commun de partager un certain nombre de présupposés :

- celui de reconnaître aux approches paradoxales en management une capacité à renouveler le regard sur les dynamiques organisationnelles (d'apprentissage, de changement, d'innovation, etc.) à rebours d'une tradition rationaliste et d'une pensée dichotomique qui a longtemps dominé la théorie des organisations. Cette perspective est également de nature à renouveler la pratique managériale pour des praticiens parfois tentés de réduire la figure du paradoxe à celle du dilemme (le choix entre deux alternatives) quand ils ne sont pas dans le déni. Or, dans le paradoxe, ce choix n'est pas possible contrairement au dilemme. La pensée paradoxale est ainsi une pensée exigeante qui implique une capacité à penser simultanément le délibéré et l'émergent, le
\end{abstract}


contrôle et l'autonomie, l'exploitation et l'exploration, la stabilité et le changement. - celui de reconnaître aux outils de gestion un rôle central dans la régulation des paradoxes organisationnels. La double dimension habilitante et contraignante des outils de gestion, leur rôle dans le processus de construction du sens comme leur capacité à focaliser l'attention des acteurs organisationnels contribuent, de fait, à inscrire les artefacts au cœur des dynamiques organisationnelles. On ajoutera qu'une des vertus liées à l'introduction de nouveaux outils de gestion est d'agir comme un révélateur des paradoxes qui traversent l'organisation, renforçant ainsi leur lisibilité et leur conscientisation par les acteurs.

Ce dossier propose de dépasser la seule qualification des paradoxes organisationnels afin d'envisager les voies de leur régulation. La littérature comme la pratique managériale, de ce point de vue, donnent à voir la prégnance de modes défensifs de gestion des paradoxes. Ces derniers ont été notamment détaillés dans l'article fondateur de Poole et Van de Ven (1989) et repris dans les travaux récents de Smith et Lewis (2011) ou bien encore Jarzabkowski et al. (2013).

Le premier mode défensif de gestion des paradoxes réside dans le déni, cette myopie organisationnelle étant susceptible d'être accentuée par les routines défensives que mobilisent les acteurs (notamment la volonté d'apparaître comme décideur rationnel). La régression peut être considérée comme une variante du déni dans la mesure où elle marque une tentative de retour à une situation antérieure où la perception de tensions contradictoires était nettement moins aiguë. On observera toutefois que ni le déni ni la régression n'aboutissent à dissoudre le paradoxe qui reste ainsi à l'état latent.
La séparation, pour sa part, se veut une réponse non conflictuelle puisqu'elle prend soin d'éviter la confrontation entre les deux pôles de la tension à l'œuvre en travaillant à leur cloisonnement. Ce dernier peut alors être temporel (Romanelli et Tushman, 1994) à travers l'alternance de phases privilégiant chacune un des pôles de la tension, ou spatial (Gibson et Birkinshaw, 2004) via la présence dans l'organisation d'espaces dédiés spécifiquement à l'une des polarités, ou leur localisation à différents niveaux de la structure. Le dilemme consiste à privilégier un des pôles de la tension à l'exclusion de l'autre, souvent en fonction d'un calcul rationnel bénéfices/coûts de chaque option. La gestion par le dilemme constitue une option risquée, la suppression d'un des pôles de la tension à l'œuvre créant des déséquilibres rarement soutenables à terme. La quête de compromis traduit la volonté de trouver un point d'équilibre entre les deux pôles via un jeu de concessions réciproques qui, finalement, risque de conduire à leur affaiblissement mutuel (Bollecker et Nobre, 2016).

Les effets délétères de ces modes défensifs sont désormais bien documentés. En premier lieu, on observera qu'aucun ne répond réellement à la définition du paradoxe entendu comme l'expression de « contradictions durables, voire permanentes, entre des éléments qui apparemment s'excluent l'un l'autre mais coexistent malgré tout » (Quinn et Cameron, 1988). Ces réponses défensives, parfois mobilisées de façon routinière, ont pour finalité première, si ce n'est d'évacuer, du moins de limiter le potentiel perturbateur des paradoxes.

Les réponses managériales actives, celles qui s'efforcent de reconnaître le potentiel créatif des paradoxes plutôt que de chercher 
à les dissoudre, sont à la fois rares et plus exigeantes. La confrontation (Poole et Van de Ven, 1989 ; Lewis, 2000) consiste à préserver voire entretenir délibérément les deux pôles de la tension et à gérer les conflits nés de leur rencontre à travers le débat et la création d'espaces de discussion autour des paradoxes. L'ajustement (Jarzabkowski et al., 2013) reconnaît la légitimité de chaque pôle de la tension ainsi que leur interdépendance. Les deux doivent ainsi être simultanément adressés dans une refonte des pratiques managériales. La transcendance (Gibbs, 2009 ; Smith et al., 2010 ; Smith et Lewis, 2011 ; Clegg et Pina e Cunha, 2017) s'affronte au paradoxe en l'intégrant dans un nouveau cadre de référence, la production d'une nouvelle synthèse (qui peut s'incarner dans un concept tiers et médiateur) qui efface la controverse précédente et inscrit l'organisation dans une dynamique d'apprentissage. Cette diversité des modes de prise en charge des paradoxes, passifs comme actifs, suggère leur combinaison possible sur la durée, afin d'ajuster les réponses, managériales en fonction de la nature des paradoxes à l'œuvre, ainsi qu'aux acteurs engagés dans leur régulation. De ce point de vue, les textes rassemblés dans ce dossier appellent à une nécessaire contextualisation dans la façon d'appréhender les paradoxes, en montrant le poids des pressions institutionnelles mais aussi de la culture organisationnelle ou bien encore des ressources à disposition dans le management des paradoxes (Guilmot et Ehnert, 2015).

C'est ces enjeux que donnent à voir l'article de Caroline Tahar. À travers une recherche intervention déployée dans un CROUS, elle analyse le paradoxe né de la volonté de cet établissement à but non lucratif d'améliorer la rentabilité de ses activités marchandes, sans pour autant mettre en péril, voire en favorisant ses missions sociales. La stratégie paradoxale mise en œuvre pour gérer cette tension contradictoire combine ainsi plusieurs réponses managériales : la séparation temporelle pour l'activité d'hébergement qui intègre judicieusement la temporalité spécifique de cette activité, la confrontation pour l'activité restauration avec la création de nouvelles formules en complément au tarif social (d'une certaine manière le paradoxe est externalisé sur le bénéficiaire du service qui le résout par son choix de consommation), la synthèse à travers la prestation mobilité qui concilie d'apporter une meilleure réponse à un besoin social tout en générant de nouvelles sources de revenus.

Cette diversité des modes de réponse est également à l'œuvre dans la contribution de Vichara Kin, Nathalie Fabbe-Costes et Frédéric Prevot qui propose une relecture de la tension entre apprentissage par exploitation et apprentissage par exploration en contexte interorganisationnel, celui des alliances stratégiques. Elle repère trois modes principaux de gestion de ce paradoxe activés par les managers d'alliances, deux relevant d'une gestion par le dilemme (l'exploitation au détriment de l'exploration et vice-versa), le troisième s'efforçant de réconcilier les deux polarités via le recours à l'ambidextrie. Pour leur part, Maël Sommer, Karine Gauche et Leïla Temri analysent le cas de petites entreprises dans le milieu agricole. Ce secteur se caractérise par des crises récurrentes et des évolutions profondes. Dans cet environnement complexe, certaines petites entreprises développent des «stratégies collectives ». Celles-ci leur permettent de résoudre les tensions inhérentes à leur situation, constituent un mode 
de management des paradoxes et assurent un management durable dans ces organisations.

Par ailleurs, la quête d'une perspective plus dynamique et intégrée sur les paradoxes organisationnels peut difficilement faire l'impasse d'une perspective multi-niveaux, les paradoxes étant susceptibles de se cristalliser tant dans un registre individuel, que collectif, organisationnel voire interorganisationnel. La co-évolution des paradoxes à différents niveaux devient alors un enjeu pour la recherche (Jarzabkowski et al., 2013). C'est cette perspective d'une approche paradoxale multiniveaux que mobilisent Anne-Sophie Thelisson, Olivier Meier, Audrey Missonier et Gilles Guieu pour analyser les conditions d'une intégration post-fusion. L'article s'appuie pour ce faire sur une étude de cas longitudinale en temps réel relative à la fusion de deux sociétés immobilières d'investissement parisiennes cotées. L'analyse du processus d'intégration post-fusion montre que les paradoxes qu'il cristallise sont gérés par une démarche ellemême paradoxale combinant gestion par le dilemme et hybridation organisationnelle. Trouver des voies innovantes pour gérer les paradoxes passe sans doute par l'adoption de cadres d'analyse qui, précisément, rendent justice à cette notion de paradoxe. C'est l'ambition de l'article de Khoudia Guèye et Elise Marcandella qui explore, à l'aide d'une étude de cas longitudinale - le cas SIRUS - les tensions et paradoxes générés dans les phases amont de projets innovants collaboratifs (PIC), en l'occurrence ceux s'inscrivant dans le cadre de pôles de compétitivité. Les auteures mobilisent un cadre théorique original, celui de la théorie des systèmes d'activités, qui reconnaît un rôle central aux instruments dans la dynamique de l'action collective mais aussi aux logiques communautaires, aux institutions (règles) ainsi qu'aux structures (division du travail).

L'article de Justine Arnoud, Cathy Krohmer et Pierre Falzon explore lui aussi la capacité de la théorie des systèmes d'activité à rendre compte des effets des paradoxes dans les organisations et à proposer des modes de régulations de ceux-ci. À partir du cas d'un centre de service partagé qui peut être caractérisé comme étant une « organisation paradoxante », c'est-à-dire une organisation qui applique une gestion défensive des paradoxes, en l'occurrence le déni (les appels téléphoniques sont interdits mais tout le monde « sait » qu'ils existent), cet article montre que la théorie des systèmes d'activité permet d'identifier les contradictions qui émergent dans le travail lui-même et de développer sur celles-ci une discussion, une re-construction collective du sens et ainsi une régulation qui permet d'éviter les effets délétères des paradoxes.

\section{BIBLIOGRAPHIE}

Bollecker G. et Nobre T. (2016). « Les stratégies de gestion des paradoxes par les managers de proximité : une étude de cas », Recherches en Sciences de Gestion, no 113, p. 43-62.

Clegg S.R. et Pina e Cunha M. (2017). "Organizational dialectic”, The Oxford Handbook of Organizational Paradoxes, W.E. Smith, M.W. Lewis P. Jarzabkowski et A. Langley (Dir), Oxford University Press, Oxford, UK, p. 105-124 
Gibbs J. (2009). "Dialectics in a global software team: Negociating tensions across time, space and culture", Human Relations, vol. 62, n 6, p. 905-935.

Gibson C.B. et Birkinshaw J. (2004). "The antecedents, consequences and mediating role of organizational ambidexterity”, Academy of Management Journal, vol. 47, n 2, p. 209-226.

Guilmot N. et Ehnert I. (2015). "27 years of research on organizational paradox and coping strategies: A review", Actes de la XXIV Conférence de l'AIMS, Paris, 3-5 juin.

Jarzabkowski P., Lê J.K. et Van de Ven A.H. (2013). "Responding to Competing strategic demands: How organizing, belonging, and performing paradoxes coevolve", Strategic Organization, vol. $11 \mathrm{n}^{\mathrm{o}} 3$, p. 245-280.

Lewis M.W. (2000). “ Exploring paradox: Toward a more comprehensive guide”, The Academy of Management Review, vol. 25, $\mathrm{n}^{\mathrm{o}}$ 4, p. 760-776.

Quinn R. et Cameron K. (1988). Paradox and transformation: Toward a theory of change in organization and management, Ballinger, Cambridge, MA.

Romanelli E. et Tushman M.L. (1994). "Organizational transformation as punctuated equilibrium: An empirical test", Academy of Management Journal, vol. 37, n 5, p. 11411166.

Smith W.K. et Lewis M.W. (2011). "Toward a theory of paradox: A dynamic equilibrium model of organizing", Academy of Management Review, vol. 36, $\mathrm{n}^{\circ}$ 2, p. 381-403.

Smith W.K., Binns A. et Tushman M.L. (2010). "Complex business models: Managing strategic paradoxes simultaneously", Long Range Planning, vol. 43, n 2, p. 448-461. 